\title{
NONLINEAR DYNAMICS OF BUCK CONVERTER
}

\author{
Dmitry Pikulin \\ Riga Technical University, Faculty of Electronics and Telecommunications \\ Azenes 12, Riga, Latvia \\ Ph.: +(371)26565214, e-mail: pikulin03@inbox.lv
}

\begin{abstract}
This paper is concerned with the problem of modeling and simulation of piecewise linear systems with the pulse width modulation control. Models of this kind of systems are widely used in engineering practice, in particular in power electronic converters. The research provides the study of chaos and bifurcations in one of the switching converters - step-down converter under voltage mode control, operating in discontinuous and continuous current modes, using Matlab and Simulink simulation environment. Various types of models, including discrete - time maps, switched state - space models are examined in order to detect their suitability and reliability in numerical investigation of nonlinear phenomena in DC-DC converters. Some analytical methods for the search of periodic regimes and their stability estimation were also used in order to validate numerically obtained results and evaluate the accuracy of models used. As direct simulation does not accurately pinpoint bifurcation points and computes only stable invariant sets, some aspects and advantages of numerical path-following are also discussed.
\end{abstract}

Keywords: bifurcation diagrams, buck converter, chaos, path-following, simulation tools.

\section{Introduction}

High efficiency solid state power conversion has become possible through the continuing development of high power semiconductor devices. The operation of these devices as switches, which is necessary for high efficiency, means that power electronic circuits are essentially nonlinear, time varying dynamical systems. Thought this makes them difficult to study, the effort is well worthwhile as this systems are becoming increasingly important in the delivery of electrical energy.

In the case of switch mode power converters (SMPC) the most commonly used models are: averaged model (AM), switched state-space model (SSSM) and iterative mapping (IM). A considerable amount of work in analysis of nonlinear phenomena in DC-DC power converters has been reported during the latest three decades [1-4]. It has been proved that the use of AM, commonly accepted in engineering practice, does not allow determination of the existence of the majority of nonlinear phenomena in SMPC. Moreover, Tse in his study [5] showed that the conventional analysis using averaging is qualitatively wrong: it predicts stability in large parameter range, whereas in reality higher order orbits and chaos are present. Therefore, rather than recurring to AM, which are standard tools for the analysis of this kind of circuits, in our investigation we provide the analysis of the bifurcation phenomena in SMPS, highlighting regions where subharmonic and even aperiodic behavior is possible, using the most suitable nonlinear models (SSSM and IM).

\section{Materials and methods}

Step-down (buck) switching converter is one of the most popular circuits in modern electronics. It is especially widely used in the field of handheld devices that require many different voltage levels to be supplied by the same battery with minimal waste of energy. Among many possible implementations of this converter we focus on the pulse-width modulated (PWM) buck DC-DC converter with voltage mode control, whose circuit is schematized in Fig.1. 


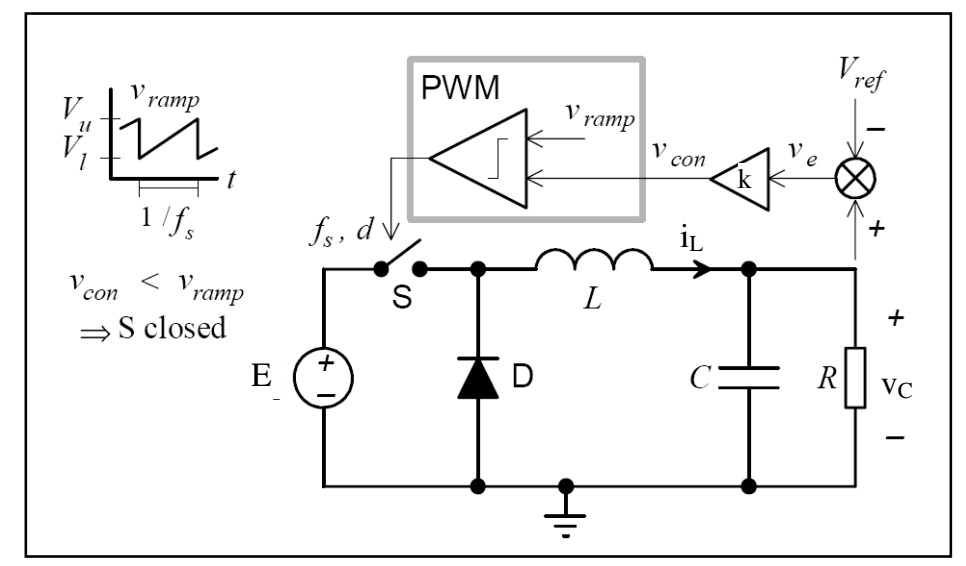

Fig. 1. PWM buck converter under voltage mode control

The aim of buck converter is to preserve preferred output voltage lower than that provided by the input source. This can be achieved by appropriately turning on and off the switch, so that the circuit is repeatedly forced by the external voltage source. The SSSM consists of three linear susbsystems depending on the value of inductor current and switching condition - the value of the output voltage is compared with the ramp signal to generate the switching signal.

$$
\begin{aligned}
& \left\{\begin{array}{l}
\frac{d v_{C}}{d t}=\frac{1}{C}\left(i_{L}-\frac{v_{C}}{R}\right) \text { for } v_{C} \geq \frac{V_{\text {ramp }}}{k}+V_{\text {ref }} ; \\
\frac{d i_{L}}{d t}=\frac{1}{L}\left(-v_{C}\right)
\end{array}\right. \\
& \left\{\begin{array}{l}
\frac{d v_{C}}{d t}=\frac{1}{C}\left(i_{L}-\frac{v_{C}}{R}\right) \text { for } \quad v_{C}<\frac{V_{\text {ramp }}}{k}+V_{\text {ref }} \text { and } i_{L}>0 ; \\
\frac{d i_{L}}{d t}=\frac{1}{L}\left(E-v_{C}\right)
\end{array}\right. \\
& \left\{\begin{array}{l}
\frac{d v_{C}}{d t}=\frac{1}{C}\left(-\frac{v_{C}}{R}\right) \text { for } \quad v_{C}<\frac{V_{\text {ramp }}}{k}+V_{\text {ref }} \text { and } i_{\mathrm{L}}=0 ; \\
\frac{d i_{L}}{d t}=0
\end{array}\right. \\
& V_{\text {ramp }}=V_{L}+\left(V_{U}-V_{L}\right)\left(\frac{t}{T} \bmod 1\right)
\end{aligned}
$$

where $\mathrm{v}_{\mathrm{C}}$ corresponds to output voltage; $\mathrm{i}_{\mathrm{L}}$ - inductor current; $\mathrm{k}$ is the gain of the error amplifier; $\mathrm{V}_{\text {ref }}$ - reference voltage; $\mathrm{V}_{\text {ramp }}$ - is an external ramp signal with period $\mathrm{T}$ and upper and lower threshold voltages $\mathrm{V}_{\mathrm{U}}$ and $\mathrm{V}_{\mathrm{L}}$, respectively, that is used to determine the switching instants; C,E,L and $\mathrm{R}$ are positive constants representing the capacitance, input source voltage, inductance and resistance, respectively.

Typically in high-power applications, where current ripple must be small to minimize losses, the components and threshold signal are chosen so that the inductor current is always strictly positive - the circuit is said to work in continuous conduction mode (CCM) and SSSM includes equations (1), (2), (4). On the other hand, for low-power applications, where opening and closing of switches causes a significant amount of overall losses, lower switching frequencies are used, letting the inductor current go to zero - the converter is operating in discontinuous conduction mode (DCM) and SSSM includes equations (1)-(4).

The best way to study the dynamics of the buck converter is to obtain a summary chart of the different types of behavior exhibited by circuit when some parameters are varied. For piecewise smooth systems there are two classes of numerical methods for investigating the possible dynamics for the range of parameters, namely: brute force approach and numerical continuation (also known as path-following) [6]. 
In order to determine stable behavior, direct numerical simulation methods can be used in an event-driven manner (brute-force approach). That is, for a given initial state, the equation (1) is solved exactly or via high - order time-stepping scheme such as Runge-Kutta method. The test function is monitored in order to determine the next switching point. After applying the switching law, this point is then used as the starting point for the next calculation (solving equation (2) or (3)). This process could be easily applied to calculate flows with many switching events or points of IM. To determine possible sets for particular parameter value a random set of initial data is chosen and the orbits from each such point are calculated over several hundreds periods (or iterations in case of IM) without storing, than the flow is continued for another hundred periods and stored. To see how the set changes, a small parameter adjustment is made and the process is repeated. The solution data obtained in such a manner can then be plotted in a bifurcation diagram where solutions are plotted against the parameter. Plots obtained in this way via direct numerical simulations are sometimes referred as Monte Carlo (or brute-force) bifurcation diagrams. This method has the advantage of capturing most (but not all) of the long time dynamics, but has the disadvantage of not being able to capture unstable behavior, which requires another algorithm, called path-following.

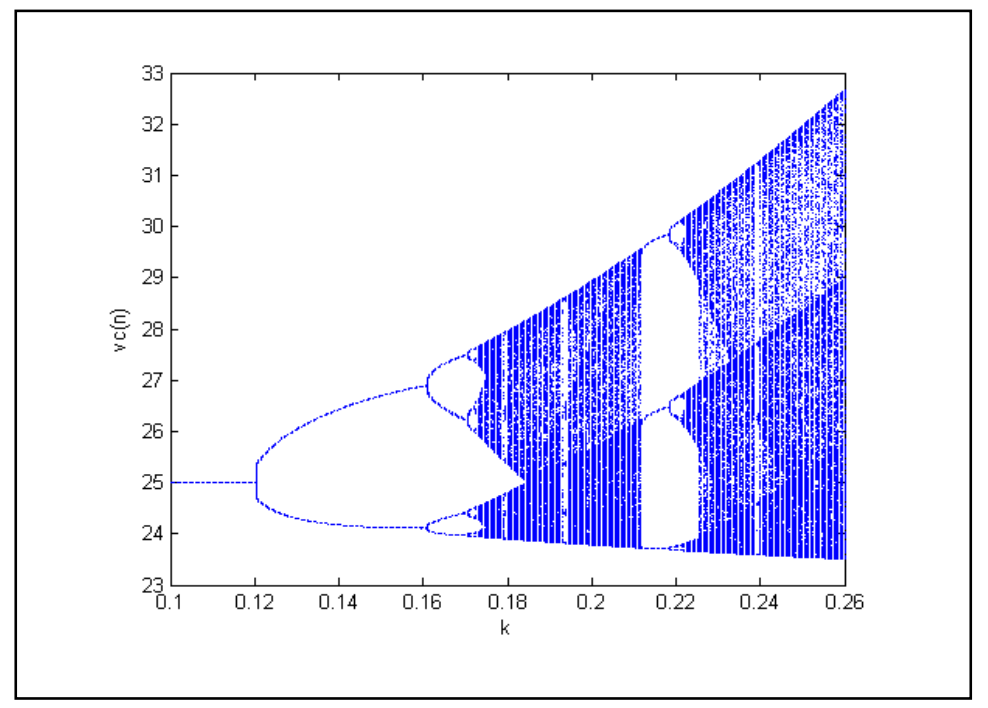

Fig.2. Monte Carlo bifurcation diagram for buck in DCM mode

In order to plot the brute-force bifurcation diagram it is necessary to calculate the Poincare section for the systems under investigation. In this case the most suitable would be the use of IM. It appears that it is not possible to obtain the closed form IM for the buck converter operating in CCM, as finding the switching instants in this case involves solving of transcendental equations. However, the operation of buck converter in DCM (when $\mathrm{v}_{\mathrm{C}}$ is the only state variable) was studied by Tse [7], who obtained discrete time one dimensional map:

$$
\begin{gathered}
v_{C, n+1}=\frac{\alpha v^{2}{ }_{C, n}+E\left(E-v_{C, n}\right) \beta\left(H\left(D-k\left(v_{C, n}-V_{r e f}\right)\right)\right)^{2}}{v_{C, n}}, \\
D=\sqrt{\frac{(1-\alpha) V_{C}^{2}}{\beta E\left(E-V_{C}\right)}} ; \alpha=1-\frac{T}{R C}+\frac{T^{2}}{2 C^{2} R^{2}} ; \beta=\frac{T^{2}}{2 L C} ; H(x)=\left\{\begin{array}{l}
0,{ }_{-} \text {if } \_x<0 \\
1, \text { if } x>1 \\
x, \text { otherwise. }
\end{array} .\right.
\end{gathered}
$$

Where $\mathrm{T}$ is the switching period, $\mathrm{V}_{\mathrm{C}}$ - steady-state output voltage and all other parameters are depicted on the Fig.1. The example of Monte Carlo bifurcation diagram obtained on the basis of this map is shown in the Fig.2. The parameters used are as follows: $\mathrm{T}=333.3(\mu \mathrm{s})$; $\mathrm{R}=12.5(\Omega) ; \mathrm{L}=208(\mu \mathrm{H}) ; \mathrm{C}=222(\mu \mathrm{F}) ; \mathrm{V}_{\text {ref }}=25(\mathrm{~V}) ; \mathrm{E}=33(\mathrm{~V})$. Small signal feedback gain was 
chosen as the bifurcation parameter. This diagram clearly shows that the period-doubling route to chaos is initiated as $\mathrm{k}$ reaches 0.12 .

While having the merits described above, direct simulation suffers from two main disadvantages - it does not accurately pinpoint bifurcation points and computes only stable periodic orbits. In order to accurately locate bifurcations it is sometimes necessary to compute unstable orbits. These typically comprise methods for numerical path-following of the solutions as parameter varies, for detecting codimension-one bifurcations, and possibly continuation of these bifurcation points in two or more parameters.

The key idea behind numerical continuation is to compute sequences of points at small intervals along the solutions curve [8,9]. The most commonly used method to compute sequences of fixed points is Newton-Raphson method, but it is known that this requires a sufficiently good initial guess in order to converge. There exists a variety of modifications of this method, but the strength of approach is the ability to follow unstable periodic orbits and to detect regimes skipped in the process of construction of Monte Carlo bifurcation diagrams. It is important to note, that when computing solutions to piecewise - smooth systems, it is usually not possible to use general purpose nonlinear software packages (such as AUTO [10], MATHCONT[11] etc.), as most numerical integration routines assume high degree of smoothness of the solution. Accurate computations must take special allowance for the nonsmooth events that occur when switching boundary is crossed. Hence, using the conditions given above, special numerical continuation routines were written in MATLAB to trace the bifurcation curves in one parameter space.

Fig.3. shows the example bifurcation diagram of the buck converter operating in DCM, computed on the basis of IM (5) by means of path-following approach.

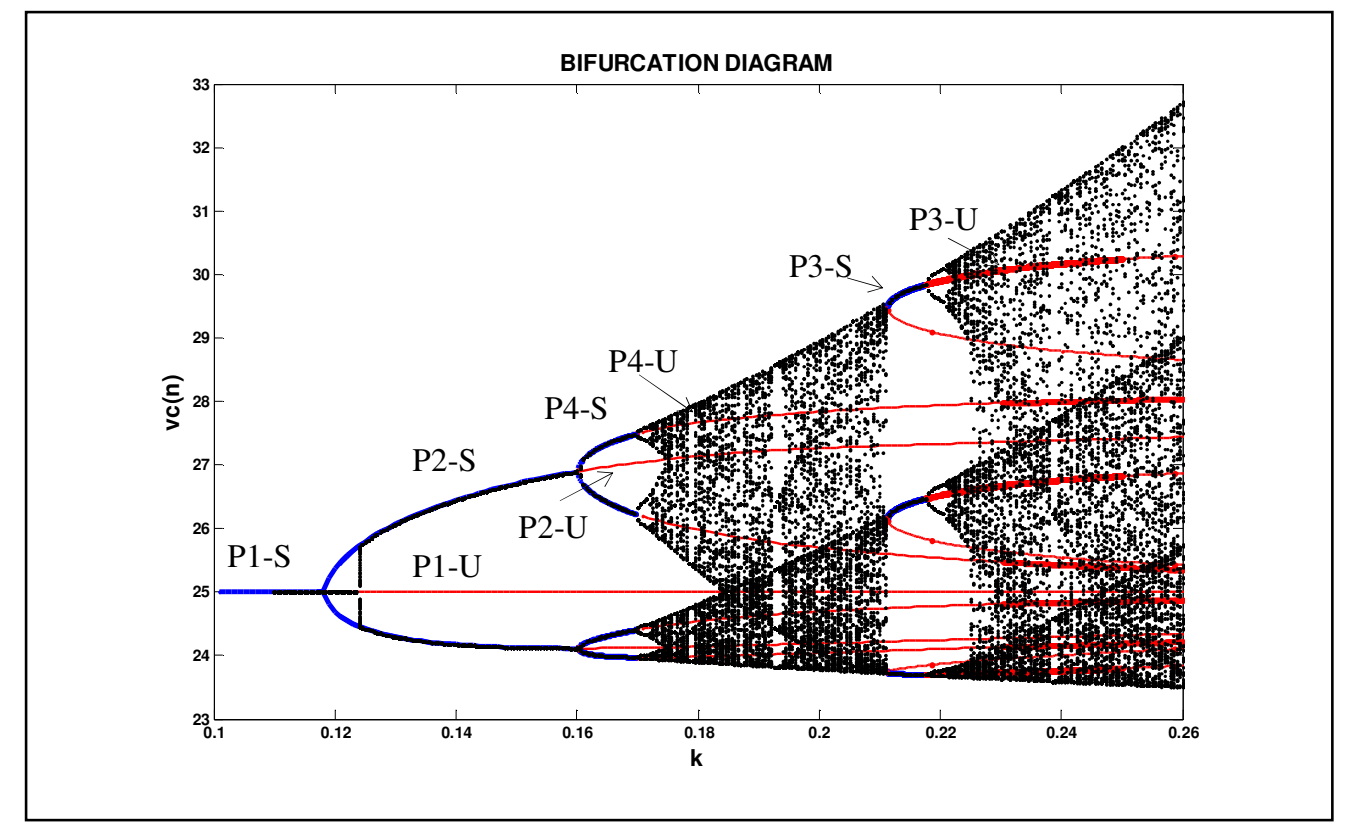

Fig.3. Bifurcation diagram for buck in DCM mode

For the sake of simplicity, this diagram depicts bifurcation branches of just few operating regimes. Blue lines represent stable orbits, thick red lines represent unstable periodic orbits (period-1 orbit is marked as P1, period-2 as P2 etc.), and black dots illustrate the brute-force diagram. It could be seen, that numerical continuation approach allows the detection of all possible periodic windows between chaotic bands as well it gives a possibility to explain some nonlinear phenomena. For example, the sudden birth of wide band chaos from several chaotic bands at $\mathrm{k}=0.223$ could be explained by collision of chaotic attractor with unstable 
period-3 orbit. This could be clearly seen on Fig.3., however, Monte Carlo bifurcation diagram does not show the explanation of this expansion of chaotic attractor.

As it was already mentioned, the path-following algorithm also uses the Poincare section of the flow, but authors established, that in the case of path-following approach, there exist some special requirements to the "quality" of this section.

The Fig.4.a. represents the phase portrait obtained for the period-1 operation of the buck converter, solving equations (1)-(4) by means of Runge - Kutta method. The solid blue line shows the exact solution of equations, and the black dashed lines - numerical solution. If this phase portrait is used to construct the Poincare section (Fig.4.b.), instead of one equilibrium point (big blue cross), corresponding to the period-1 operation, it will show a variety of points (small black crosses) around it.

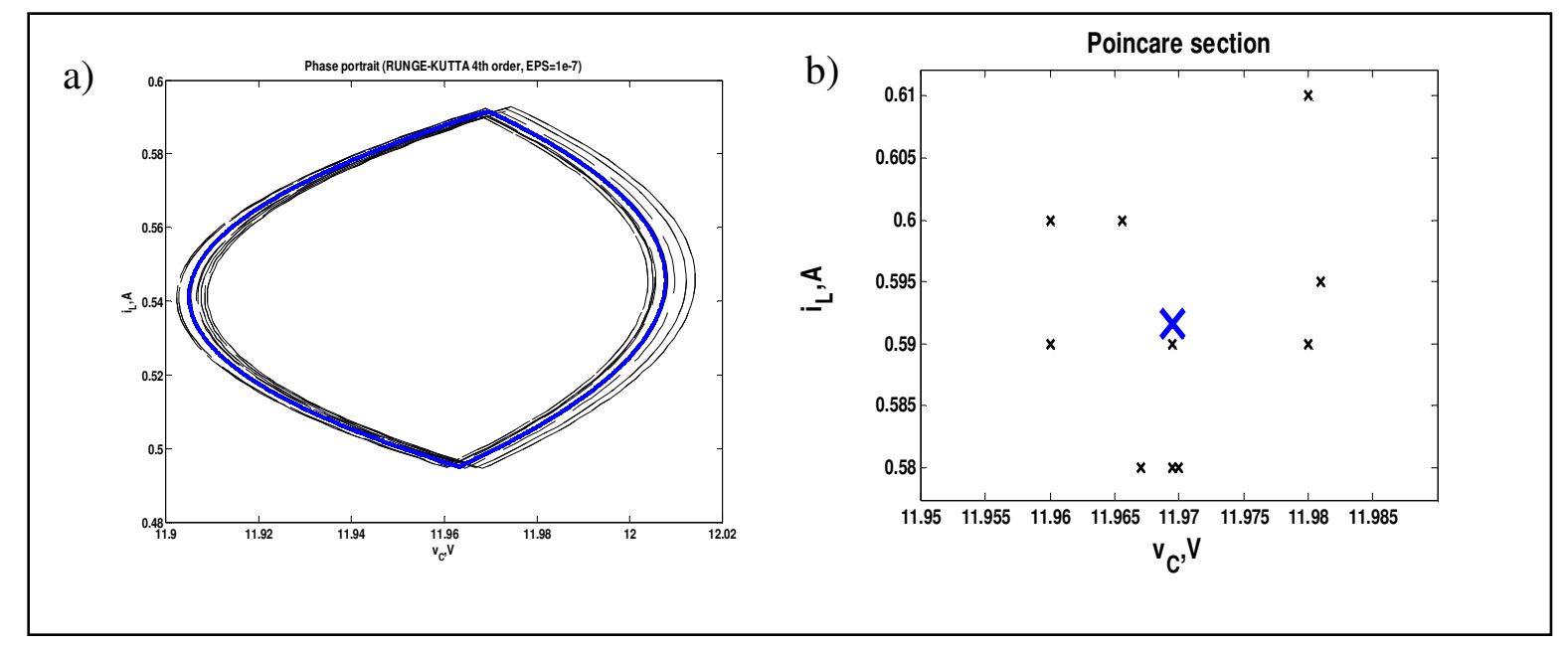

Fig.4. Period-1 phase portrait and Poincare section

Constructing the brute-force bifurcation diagram using this kind of data will lead to slightly "spread" but still sufficiently clear diagrams, similar to those, obtained in laboratory experiments in the presence of unavoidable noise. On the other hand, using the path-following algorithm, the Newton-Raphson or similar methods would not converge or will erroneously detect period-n $(n>1)$ operation regime. So in the case of numerical continuation some special modifications of the model should be used.

The main cause of the "spread" phase portrait is that the use of numerical integration with fixed step does not allow precise detection of switching events, which leads to the fact, that even in the period- 1 regime at the beginning of every cycle different starting points for the next calculations are used. We propose some useful methods, used by author in order to overcome this problem. The most straightforward approach is to decrease the overall integration step until the error becomes negligible. As the main drawback of the mentioned method is the unacceptable increase of simulation time and number of data points, the author would suggest using some numerical integration algorithms with variable step size in order to decrease the step only in the vicinity of switching event. Thus, appropriately choosing integration steps, it is possible to obtain clear Poincare sections and apply path following approach.

\section{Results and discussion}

Various types of models of the buck converter operating in CCM and DCM have been studied in order to detect their suitability and reliability in numerical investigation of nonlinear phenomena. The comparison of models is presented in the Table. As it was already 
mentioned, all models could be used in order to detect subharmonics and chaos in DC-DC converters, however it is extremely important to acknowledge the possibilities and limitations of the use of these nonlinear models.

\section{The comparison of models}

Table

\begin{tabular}{|l|l|}
\hline Model & Comments \\
\hline $\begin{array}{l}\text { SSSM } \\
\text { (analytically solved) }\end{array}$ & $\begin{array}{l}\text { + Fast and precise models; } \\
\text {-Could be applied in case of relatively simple systems of } \\
\text { linear differential equations. }\end{array}$ \\
\hline $\begin{array}{l}\text { SSSM } \\
\text { (numerical } \\
\text { integration) }\end{array}$ & $\begin{array}{l}\text { +No need to solve equations by hand; } \\
\text {-The slowest models; } \\
\text { - In order to use for numerical path following requires some } \\
\text { special particularization measures. }\end{array}$ \\
\hline Iterative mapping & $\begin{array}{l}\text { + Direct use in brute-force approach and path following; } \\
\text { +Simple and fast models; } \\
\text { - Impossible to use for buck converter operating in CCM; } \\
\text { - Requires simplification procedures - some useful } \\
\text { information could be lost. }\end{array}$ \\
\hline
\end{tabular}

It could be seen from the Table that the most attractive from the practical point of few is the IM, however, it has several noticeable drawbacks, that limit the applicability of this kind of models. In order to investigate the dynamics of buck converter, operating in CCM, we still have to use the SSSM. The example given in this paper and illustrated in the Fig.3. shows, that the use of numerical path following technique gives the most full understanding of nonlinear behavior of dynamical systems and allows explaining some complex phenomena. So the next step in this kind of research would be the development of systematic procedure, capable of merging the SSSM models of DC-DC converters and numerical path-following techniques in order to create the complete bifurcation diagrams, indicating all possible operating regimes.

\section{Conclusions}

This paper is concerned with the problem of modeling and simulation of piecewise linear systems with PWM control. The research provides the study of buck converter under voltage mode control, operating in DCM and CCM, using Matlab and Simulink simulation environment. Various types of models, including IM and SSSM, are examined in order to detect their suitability and reliability in numerical investigation of nonlinear phenomena in DC-DC converters. The main advantages and disadvantages of different models are pinpointed and some recommendations for the most appropriate use and implementations are provided. It has been shown that direct simulation does not accurately locate bifurcation points and computes only stable invariant set, so some aspects and advantages of numerical path-following are also discussed. The complete bifurcation diagrams, obtained by means of numerical continuation could be used not only to predict the operating regimes in given parameter range, but could also provide useful information for different nonlinear control schemes, when it is necessary to find and stabilize/destabilize certain periodic orbits (this technique is widely used in chaotic control [12] and spread spectrum applications[13,14]). 


\section{Acknowledgment}

This work has been supported by the European Social Fund within the project «Support for the implementation of doctoral studies at Riga Technical University».

\section{References}

1. di.Bernardo, M., Fosas, E., Olivar, G., Vasca, F. Secondary bifurcations and high-periodic orbits in voltage controlled buck converter // International Journal of Bifurcation and Chaos. - Vol.12, no.7 (1997), p.27552771.

2. Fosas, E., Olivar, G. Study of chaos in buck converter. IEEE Transactions on Circuits and Systems Part I. Vol.43, no.1 (1996), p. 13-25.

3. Hammil, D.C., Deane, J.H.B., Jefferies, D.J. Modeling of chaotic dc/dc converters by iterative nonlinear mappings. IEEE Transactions on Circuits and Systems Part I. - Vol.35, no.8 (1992), p. 25-36.

4. Chan, W.C.Y., Tse, C.K. A universal bifurcation path conjectured from current-programmed switching converters . Proceedings of International Symposium on Nonlinear Theory and Its Applications, (NOLTA'96), Japan, 1996, p.121-124.

5. Tse, C.K. Complex Behavior of Switching Power Converters. Boca Raton, FL: CRC Press, 2003, 262p.

6. di Bernardo, M., Budd, C.J., Champneys, A.R., Kowalczyk P. Piecewise-Smooth Dynamical Systems: Theory and Applications. Springer-Verlag, Berlin, 2008, 483p.

7. Tse, C.K. Chaos from a Buck Switching Regulator Operating in Discontinuous Mode. International Journal of Circuit Theory and Applications, vol. 22, no. 4, July-August, 1994,p. 263-278

8. Eugene, L., Allgower and Kurt Georg. Introduction to Numerical Continuation Methods. SIAM Classics in Applied Mathematics 45. 2003, 414p.

9. Zakrzhevsky, M. New Concepts of Nonlinear Dynamics: Complete Bifurcation Groups, Protuberances, Unstable Periodic Infinitiums and Rare Attractors. Journal of Vibroengineering. - Vol.10, iss.4 (2008), p. 421-441.

10. Dercol, F. and Kuznetsov, Yu. A. SlideCont: An Auto 97 driver for bifurcation analysis of Filippov systems. ACM Trans. Math. Software, 31 (2005), p. 95-119.

11. Dhooge, A., Govaerts, W., Kuznetsov, Yu.A. Matcont: A matlab package for numerical bifurcation analysis of odes. ACM TOMS., 29 (2003), p. 141-164.

12. Verghese, G.C. Dynamic modeling and control in power electronics. The Control Handbook / Levine, W.S. - FL: CRC Press-IEEE Press, 1996, p. 1413-1423.

13. Deane, J.H.B., Hamill, D.C.“Improvement of power supply EMC by chaos," Electron. Lett., vol. 32, no. 12, Jun. 1996, p. 1045-1049.

14. Banerjee, S., Kastha, D., SenGupta, S. Minimising EMI problems with chaos. Proc. Of Int. Conf. Electromagn. Interference Compat., Feb. 2002, p. 162-167. 